Aim of the study: F-18 FDG PET/CT is the most effective method for demonstrating extrapulmonary metastases of lung cancer. The aim of this study is to investigate the level of muscular metastasis of non-small-cell lung carcinoma (NSCLC) in F-18 FDG PET/CT and to demonstrate the characteristics of this special group of patients.

Material and methods: A total of 1150 patients with the diagnosis of NSCLC, who were referred for F-18 FDG PET/CT, were included into the study. Among these patients, the findings of 13 who were shown to have muscle metastases were studied.

Results: In the retrospective analysis of the patients, 13 patients (12 male, 1 female; mean: $59 \pm 7$ years old) were found to have haematogenous (excluding local invasion) muscular metastases of NSCLC using F-18 FDG PET/CT. Two of the 13 patients had only muscular metastases (one patient isolated, one patient two foci). The other 11 patients had additional distant metastases in six metastatic sites (bone in 7 patients, distant lymph node in 6 , adrenal gland in 5, contralateral lung in 3 , liver in 1 , and brain metastasis in 1 patient). Five patients died during the mean $11 \pm 8$ months follow up. Conclusions: Muscular metastasis is not a rare condition, especially in $\mathrm{F}-18$ FDG PET/CT examinations, and is frequently associated with additional distant metastases.

Key words: lung cancer, F-18 FDG $\mathrm{PET} / \mathrm{CT}$, muscular metastasis.

Contemp Oncol (Pozn) 2015; 19 (3): 241-245 DOI: $10.5114 /$ wo.2014.46044

\section{Haematogenous muscular metastasis of non-small cell lung cancer in F-18 fluorodeoxyglucose positron emission tomography/ computed tomography}

\author{
Karyagar Savas ${ }^{1}$, Koc Zehra Pinar ${ }^{2}$, Karyagar Saglampinar Sevda ${ }^{1}$, \\ Kesici Ugur ${ }^{3}$, Savli Evrim ${ }^{4}$, Cinarka Halit ${ }^{5}$, Mungan Sevdegul 6 , \\ Balci Tansel Ansal ${ }^{2}$
}

${ }^{1}$ Department of Nuclear Medicine, Kanuni Training and Research Hospital, Trabzon, Turkey

${ }^{2}$ Department of Nuclear Medicine, School of Medicine, University of Firat, Elazig, Turkey ${ }^{3}$ Department of General Surgery, School of Medicine, University of Giresun, Giresun, Turkey

${ }^{4}$ Department of Radiation Oncology, Kanuni Training and Research Hospital, Trabzon, Turkey

${ }^{5}$ Department of Respiratory Medicine, Rize Medical Faculty, RTE University, Rize, Turkey ${ }^{6}$ Department of Pathology, School of Medicine, Karadeniz Technique University, Trabzon, Turkey

\section{Introduction}

Lung cancer is a common cause of death, and since the disease process is mortal, decision making for appropriate therapy and staging is critical. F-18 FDG PET/CT is an accurate staging method that, in particular, identifies unexpected distant metastases in lung cancer patients [1]. One of these unexpected distant metastatic sites is muscular metastasis. Although there are several case reports about muscular metastases of non-small-cell lung carcinoma (NSCLC) in the literature and, additionally, in an analysis including all the solitary extrapulmonary findings in F-18 FDG PET/CT, there has been no study on patients with muscular metastases of NSCLC [2-4]. The aim of this study is to investigate the ratio and clinical characteristics of patients with muscular metastases of NSCLC.

\section{Material and methods}

Patients

A total of 1150 patients with NSCLC were included in the study. The diagnoses of all the patients with NSCLC were confirmed by histopathology (7 squamous cell carcinoma, 5 adenocarcinoma, and 1 undifferentiated large cell cancer - a total of 13 patients) and referred to our department for F-18 FDG PET/CT for staging or restaging. None of the patients had clinical suspicion or radiological demonstration of muscular metastases. Staging of the patients was based on the F-18 FDG PET/CT and brain magnetic resonance imaging (MRI) findings.

\section{Acquisition of the PET/CT}

All the patients underwent an F-18 FDG PET/CT examination. F-18 FDG PET/CT studies were carried out using an integrated PET/CT scanner, which consisted of a full-ring HI-REZ LSO PET and a six-slice CT scanner (Siemens Biograph 6; Siemens, Chicago, USA). Patients were instructed to fast for at least 6 hours before their 18F-FDG injection. Blood glucose levels were meas- 
ured before the study, and 18F-FDG injections were administered only when the plasma glucose levels were below $11.11 \mathrm{mmol} / \mathrm{l}$. Approximately 370-555 MBq of 18F-FDG (dose calculation was performed according to body weight) was injected into the patients via intravenous line. After a waiting for 60 minutes in a semi-reclined relaxed chair, the patients were imaged using an integrated PET/ CT scanner. The CT portion of the study was performed for attenuation correction purposes only, without the administration of an intravenous contrast medium. The CT was performed in the craniocaudal direction using the following parameters: $50 \mathrm{mAs}, 140 \mathrm{kV}$, and 5-mm section thickness. PET images were also acquired in the caudocranial direction, from the base of the skull to the mid-thigh, with five to eight bed positions of 3 minutes each.

\section{Image interpretation}

Two experienced nuclear medicine physicians with knowledge of the patient's clinical history interpreted the F-18 FDG PET/CT. The images were displayed in rotating maximum intensity projections and in axial, coronal, and sagittal planes. The PET-CT images were evaluated both visually and quantitatively. Every site of increased F-18 FDG uptake was classified as malignant or benign based on the localisation and intensity of the uptake. Increased F-18 FDG uptake was defined as malignant if it was observed in sites unrelated to physiological or benign processes with higher uptake compared to the surrounding tissues for distant metastases or mediastinal blood pool for mediastinal lymph node metastases. Increased F-18 FDG uptake was defined as benign if it was located in a site of known physiological distribution of the F-18 FDG or related to a known non-malignant process, such as inflammation, calcified lymph nodes, etc. Quantification of the F-18 FDG uptake was carried out by creating a region of interest over the area of maximal radiotracer activity in a primary lung tumour, mediastinal lymph nodes, and each distant lesion. Maximum standardised uptake values $\left(S_{U V} V_{\max }\right)$ were automatically generated according to the following equation:

$$
\mathrm{SUV}_{\max }(\mathrm{bw})=\text { Ctis/Dinj/bw, }
$$

where SUV ${ }_{\text {max }}(b w)$ is the maximum SUV normalised for body weight; Ctis is the tissue concentration expressed as $\mathrm{MBq}$ per millilitre; Dinj is the injected dose expressed in $\mathrm{MBq}$; and bw is the body weight expressed in kilograms. Measurement of the largest diameter and density judgment (isodense or hypodense compared to the surrounding tissues) were performed in CT images of each muscular metastasis. SUV $V_{\max }$ levels above 2.5 were considered to be malign uptake.

\section{Results}

In the retrospective analysis of the patients, thirteen patients (12 male, one female; mean: $59 \pm 7$ years old) were observed to have haematogenous (excluding local invasion) muscular metastases of NSCLC using F-18 F-18 FDG PET/CT (7 patients solitary muscular metastases and

Table 1. Clinical characteristics of the muscular metastasis

\begin{tabular}{|c|c|c|c|c|c|}
\hline $\begin{array}{l}\text { Patient } \\
\text { No. }\end{array}$ & Localization & $\begin{array}{l}\text { Lesion size } \\
\quad(\mathrm{mm})\end{array}$ & $\begin{array}{l}\text { SUV }_{\max }^{*} \\
\text { (metastasis) }\end{array}$ & $\begin{array}{l}\mathrm{SUV}_{\max }^{*} \\
\text { (tumour) }\end{array}$ & Extramuscular distant metastases \\
\hline 1 & $\begin{array}{l}\text { Internal oblique (abdominal) } \\
\text { Vastus lateralis } \\
\text { Rectus femoris }\end{array}$ & $\begin{array}{l}25 \\
10 \\
10\end{array}$ & $\begin{array}{l}11.9 \\
3.49 \\
3.34\end{array}$ & 26.1 & bone \\
\hline 2 & $\begin{array}{l}\text { Gluteus minimus } \\
\text { Semitendinosus }\end{array}$ & $\begin{array}{l}20 \\
25\end{array}$ & $\begin{array}{c}11 \\
9.5\end{array}$ & 12.5 & none \\
\hline 3 & Infraspinatus & 25 & 15.9 & 15.7 & lymph node, bone \\
\hline 4 & Gluteus minimus & 15 & 6.1 & 10.5 & lung, adrenal gland, bone \\
\hline 5 & Intercostalis muscle & 17 & 5.5 & 8.9 & lung, adrenal gland, lymph node \\
\hline 6 & $\begin{array}{l}\text { Latissimus dorsi } \\
\text { Iliopsoas } \\
\text { Gluteus maximus }\end{array}$ & $\begin{array}{c}12 \\
10 \\
8\end{array}$ & $\begin{array}{c}6.4 \\
5 \\
5.6\end{array}$ & 4.2 & lymph node, adrenal gland, bone \\
\hline 7 & $\begin{array}{l}\text { Deltoideus muscle (three foci on } \\
\text { right, one focus on left) }\end{array}$ & $\begin{array}{c}15 \\
10 \\
8 \\
20\end{array}$ & $\begin{array}{c}10 \\
7 \\
5.6 \\
7.6\end{array}$ & 15.1 & lymph node, liver, bone \\
\hline 8 & Psoas & 12 & 6.2 & 6 & none \\
\hline 9 & Iliopsoas & 15 & 16.2 & 18.4 & adrenal gland, bone \\
\hline 10 & Multiple & $5-30$ & $1.5-21.6$ & 14.9 & adrenal gland, bone \\
\hline 11 & $\begin{array}{l}\text { Deltoideus muscle } \\
\text { Quadratum lumborum }\end{array}$ & $\begin{array}{l}15 \\
24\end{array}$ & $\begin{array}{l}12.2 \\
11.4\end{array}$ & 12.2 & lymph node, adrenal gland, brain \\
\hline 12 & Erector spina muscle & 20 & 5.7 & 6.6 & lymph node \\
\hline 13 & Intercostalis muscle & 18 & 5.7 & 9.7 & lung \\
\hline
\end{tabular}


6 patients have multiple muscular metastases). The lesion size, localisation, and intensity of the lesions with primary tumour uptake are summarised in Table 1.

The mean metastasis size was $16 \pm 7 \mathrm{~mm}$, mean metastases SUV max $_{\text {mas }} 8 \pm 5$ and mean primary tumour SUV was $12 \pm 6$. Six patients had hypodense lesions, five had isodense lesions, and two patients had both isodense and hypodense lesions according to the CT characteristics of the muscular metastases.

Among the eleven patients with additional distant metastases, six metastatic sites (bone in seven patients, distant lymph node in six, adrenal gland in five, contralateral lung in three, liver in one, and brain metastasis in one) were determined. Among these thirteen patients, two had only muscular metastases (Figs. 1, 2).

Histopathological confirmation of the muscular metastases was obtained in three patients (two with isolated muscular metastases). In the mean $11 \pm 8$ month follow up, five patients died during disease course.

\section{Discussion}

According to the results of our analysis, $1 \%$ of the NSCLC patients had muscular metastases and $0.1 \%$ of the
NSCLC patients had only muscular metastases. It can be seen that the patients with muscular metastases usually had additional distant metastases, because there were just two cases (15\%) with only muscular metastasis. Histopathological confirmation was required in three patients in our group, and two of them were patients with only muscular metastases. Radiotherapy was planned for the additional muscle involvement in these three patients. The muscular metastases changed the stage and patient management in three $(23 \%)$ patients.

Common distant metastatic sites of NSCLC are bone, the adrenal glands, and the brain [3]. However, there are many unexpected metastatic sites in the F-18 FDG PET/CT examinations of patients with NSCLC. In a previous study that included 350 patients, 110 patients (31\%) were observed to have extrapulmonary metastases and $21 \%$ had solitary extrapulmonary metastases [4]. Since PET/CT is a whole body screening modality, it has the advantage of showing distant metastatic sites. Sometimes unexpected distant metastases are underestimated in routine practice, especially in unexpected sites, but further investigation of these sites is essential since nearly half of them may be malignant [4].
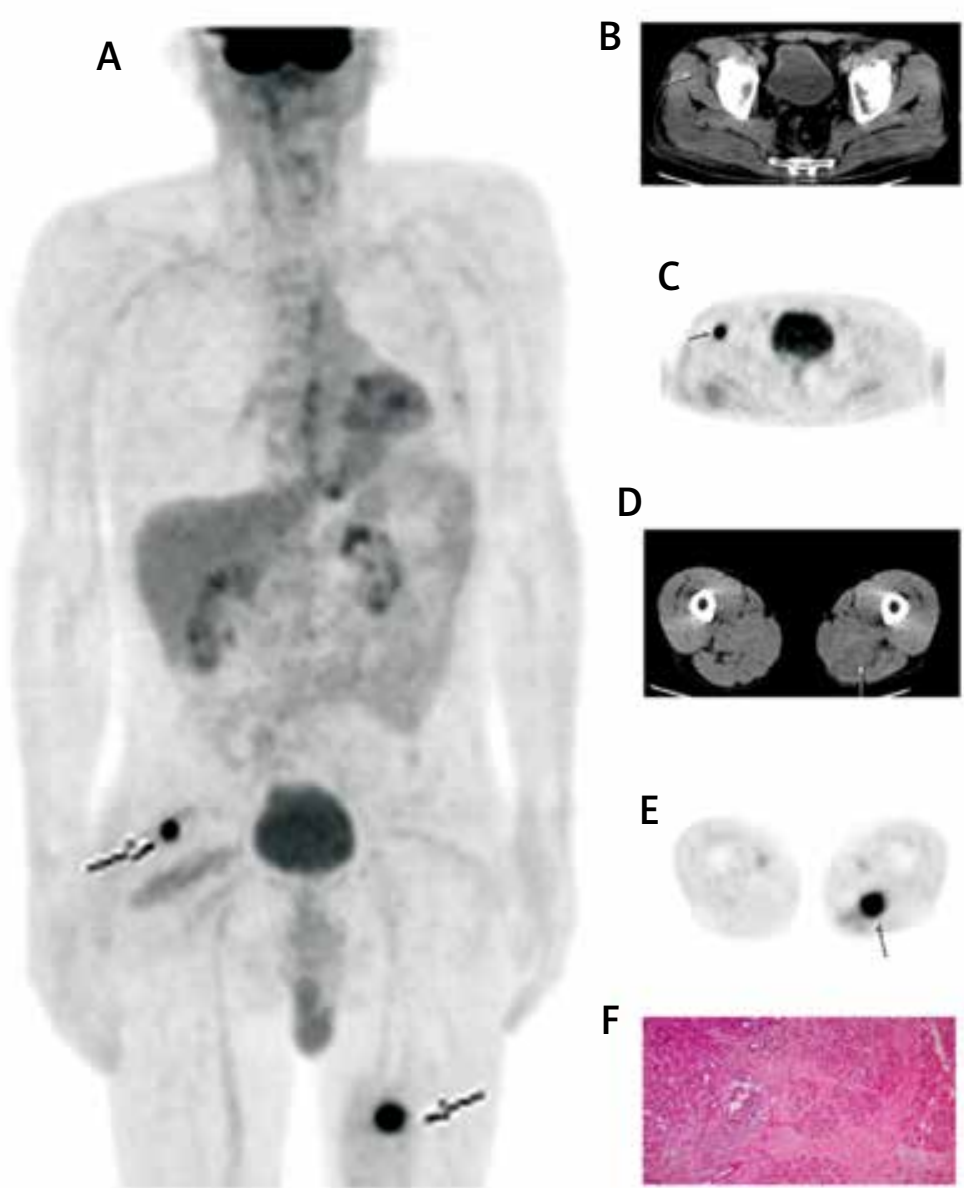

D

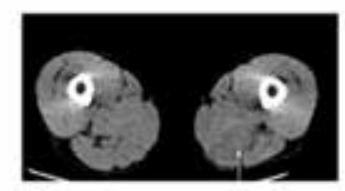

$\mathrm{E}$

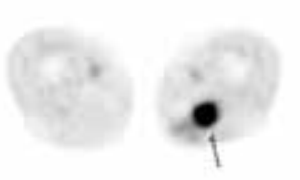

$\mathrm{F}$

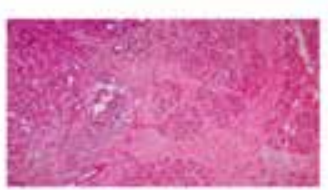

Fig. 1. Follow-up PET/CT images of a 52-year-old NSCLC patient. A) Coronal slices of PET image show two muscular metastases located in the right gluteus minimus and left semitendinosus muscles (arrow). B) Transaxial CT image of gluteus minimus metastases, and C) Transaxial PET image that corresponds to the metastases. D) Transaxial slice CT image of left semitendinosus muscle, and E) a corresponding PET image of same metastases. F) Adenocarcinoma foci that infiltrate the muscle tissue haematoxylin eosin staining $\times 100$ 


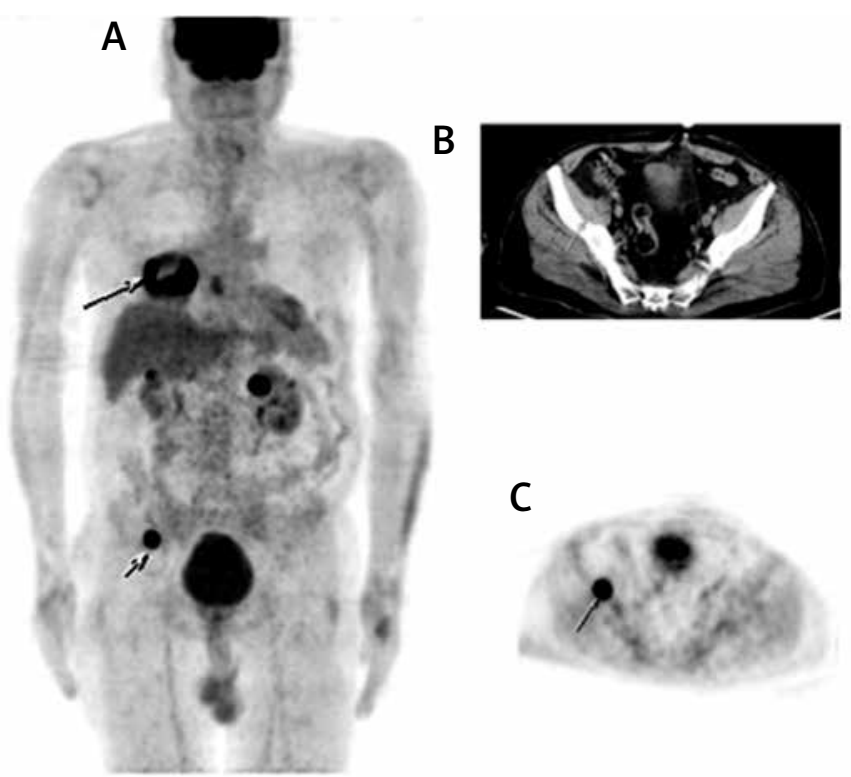

Fig. 2. PET/CT images of a 55-year-old NSCLC patient for staging. A) Coronal plane PET image of the patient depicts a primary tumour in the right lung (arrow) and left iliopsoas metastases in the muscular plane (arrow). B) Transaxial CT image of pelvis shows the right iliopsoas muscle metastasis. C) PET image of the same region

Muscular metastasis is a rare entity that has been documented to have a rate of $0.2 \%$ in an autopsy series [5]. In a series of patients with different kinds of malignancies the frequency of muscular metastases was found to be $1 \%$, and 8 cases with muscular metastases were NSCLC in that series [6]. However, Haygood et al. [7] investigated skeletal muscular metastases in their patient population of various cancers compared to the literature and claimed that this kind of metastases is not actually so rare. Although we also reported an incidence of $1 \%$, there is a series by Touheti et al. [8] in which it was reported to be $0.16 \%$. Lung cancer was the most common cancer to metastasise to muscular in their series, as in the series of Khandelwal et al. [9].

The identification of muscular metastases by $\mathrm{CT}$ is problematic because the metastatic tissue can be hypodense or isodense. Magnetic resonance imaging also cannot provide sufficient data in all cases although there are exceptional case reports [10, 11]. Nonetheless, F-18 FDG PET/CT has the advantage of being an integrated method. Sole PET or CT might underestimate muscle metastasis because the correct anatomic localisation of an F-18 FDG uptake is sometimes impossible by means of only PET. Muscular metastasis is a relatively new entity probably because identification of it depends on F-18 FDG PET/CT. Our patients also had no symptoms of muscle metastases, and no radiological imaging method has identified these lesions before PET-CT exam. Thus, if our patients did not have an F-18 FDG PET/CT examination, their muscle metastasis would not be demonstrated. Furthermore, PET/ CT-guided percutaneous biopsy of isolated muscular metastasis of another primary tumour has been reported [12].

In a recent review analysis, previous case reports with muscular metastases were analysed and it was observed that half of these metastases changed the treatment planning of the patients [13]. In our series of NSCLC patients this amounted to $23 \%$. Additional radiotherapy planning was implicated in these three patients. Treatment protocols related to muscular metastases vary in the literature. As an example, in a recent case report chemotherapy was the treatment of choice instead of surgery [3]. However, if muscular metastasis is the only metastatic site, surgical excision of both the mass and metastasis may be possible [3]. On the other hand, a patient with only one muscular metastasis is extremely rare; we had just one such patient $(0.9 \%)$ in our series. This patient received radiotherapy and died during the five-month follow up. Although there is no consensus about the therapeutic management of NSCLC patients with muscular metastases, radiotherapy of the muscular metastases is a good option, as previously reported in a case series [14]. However, in general, muscular metastasis is considered to be a feature of systemic disease [15], and systemic therapy regimens are preferred. In a previous case report on metastasectomy, surgery for primary tumour and additional systemic chemotherapy was depicted as the choice of treatment, and the case responded without relapse [10].

Among the metastatic muscles the psoas muscle is a frequent site in the literature [14-16]. In our series there were 3 patients (23\%) with psoas muscular metastases. Adenocarcinoma has been observed to be a predominant NSCLC type to metastasise to the muscular structure, according to previous case reports [14, 17, 18]. Approximately half of our patients also had adenocarcinoma metastases, but generally squamous cell type was predominant in the NSCLC group.

Muscular metastases may be asymptomatic or the initial manifestation of an unknown primary lung cancer [14, 18-20]. Haygood et al. reported 3 cases (4\%) in their study population and $70(23 \%)$ in their literature review [7]. However, most of the patients in our study were diagnosed as having primary lung cancer, and none of the patients presented with the muscular metastases first. Additionally, 
none of the patients had symptomatology relating to their muscular metastases.

The frequency of muscular metastases in the NSCLC group identified using F-18 FDG PET/CT was $1 \%$ in our patient population, which is not uncommon. F-18 FDG PET/CT might contribute to a higher percentage of patients identified as having muscular metastases. Patients with muscular metastases tend to have additional multiple metastases, so they are usually advanced stage patients.

The study was approved by the local ethics committee.

The authors declare no conflict of interests.

\section{References}

1. MacManus MP, Hicks RJ, Matthews JP, Hogg A, McKenzie AF, Wirth A, Ware RE, Ball DL. High rate of detection of unsuspected distant metastases by pet in apparent stage III non-small-cell lung cancer: implications for radical radiation therapy. Int J Radiat Oncol Biol Phys 2001; 50: 287-93.

2. Yilmaz M, Elboga U, Celen Z, Isik F, Tutar E. Multiple muscle metastases from lung cancer detected by FDG PET/CT. Clin Nucl Med 2011; 36: 245-7.

3. Purandare NC, Rangarajan V, Pramesh CS, Rajnish A, Shah S, Dua SG. Isolated asymptomatic skeletal muscle metastasis in a potentially resectable non-small cell lung cancer: detection with FDG PET-CT scanning. Cancer Imaging 2008; 8: 216-9.

4. Lardinois D, Weder W, Roudas M, von Schulthess GK, Tutic M, Moch H, Stahel RA, Steinert HC. Etiology of solitary extrapulmonary positron emission tomography and computed tomography findings in patients with lung cancer. I Clin Oncol 2005; 23: 6846-53.

5. Disibio G, French SW. Metastatic patterns of cancers: results from a large autopsy study. Arch Pathol Lab Med 2008; 132: 931-9.

6. Nguyen NC, Chaar BT, Osman MM. Prevalence and patterns of soft tissue metastasis: detection with true whole-body F-18 FDG PET/ CT. BMC Med Imaging 2007; 7: 8.

7. Haygood TM, Wong J, Lin JC, et al. Skeletal muscle metastases: a three-part study of a not-so-rare entity. Skeletal Radiol 2012; 41: 899-909.

8. Tuoheti Y, Okada K, Osanai T, Nishida J, Ehara S, Hashimoto M, Itoi E. Skeletal muscle metastases of carcinoma: a clinicopathological study of 12 cases. Jpn J Clin Oncol 2004; 34: 210-4.

9. Khandelwal AR, Takalkar AM, Lilien DL, Ravi A. Skeletal muscle metastases on FDG PET/CT imaging. Clin Nucl Med 2012; 37: 575-9.

10. Karagöz B, Bilgi O, Mahiroğulları M, et al. Lung cancer with muscle metastasis that is achieved long disease free survival by multimodal therapy: a case. Anatol J Clin Investig 2008; 2: 171-3.

11. Alhathlol A, Munk PL. Radiology for the surgeon: musculoskeletal case 44. Can J Surg 2009; 52: 246-8.

12. Mallarajapatna GJ, Kallur KG, Ramanna NK, Susheela SP, Ramachandra PG. PET/CT-guided percutaneous biopsy of isolated intramuscular metastases from postcricoid cancer. I Nucl Med Technol 2009; 37: 220-2.

13. Emmering J, Vogel WV, Stokkel MP. Muscle metastases on FDG PET-CT: a review of the literature. Nucl Med Commun 2012; 33: 117-20.

14. Strauss JB, Shah AP, Chen SS, Gielda BT, Kim AW. Psoas muscle metastases in non-small cell lung cancer. J Thorac Dis 2011; 4: 83-7.

15. Acinas García O, Fernández FA, Satué EG, Buelta L, Val-Bernal JF. Metastasis of malignant neoplasms to skeletal muscle. Rev Esp Oncol 1984; 31: 57-67.

16. Ampil FL, Lall C, Datta R. Palliative management of metastatic tumors involving the psoas muscle: case reports and review of the literature. Am J Clin Oncol 2001; 24: 313-4.
17. Kwon SY, Jeong SY, Min JJ, Oh IJ, Kim YC, Bom HS. Metastases to skeletal muscles from non-small cell lung cancer demonstrated by F18-FDG PET/CT. J Lung Cancer 2007; 6: 91.

18. Banzo I, Martínez-Rodriguez I, Quirce R, Jimenez-Bonilla J, Carril JM. Asymptomatic muscle metastasis from pulmonary adenocarcinoma. Thorax 2011; 66: 642.

19. Mogi A, Kosaka T, Yamaki E, Kuwano H. Successful resection of stage IV non-small cell lung cancer with muscle metastasis as the initial manifestation: a case report. Ann Thorac Cardiovasc Surg 2012; 18: 468-71.

20. Parajuli S, Basnet RB, Tuladhar A. Ileo-psoas muscle metastasis from pulmonary carcinoma. J Pathol Nepal 2012; 2: 251-3.

\section{Address for correspondence}

Kesici Ugur, MD, Assi. Prof.

Department of General Surgery

University of Giresun, School of Medicine

Giresun, Turkey

tel. +904543101655

$\mathrm{fax}+904543101696$

e-mail: ugurkesici77@mynet.com

Submitted: 27.01 .2014

Accepted: 20.05 .2014 